\title{
HIPERPROLACTINEMIA E DISTÚRBIOS PSIQUIÁTRICOS
}

\author{
MIRIAM C. OLIVEIRA*, CRISTINA BARDOU PIZARRO**, \\ LENARA GOLBERT ${ }^{* \star}$, CRISTINA MICHELETTO***
}

\begin{abstract}
RESUMO - Sintomas psicológicos, especialmente ansiedade e depressão, têm sido associados à hiperprolactinemia. Para avaliar a presença desses sintomas, foram submetidos à entrevista através do Composed International Diagnostic Interview, seguido pela escala de Hamilton para depressão, 32 pacientes (5 homens e 27 mulheres) com hiperprolactinemia de várias etiologias e 16 normoprolactinêmicos. A prolactina sérica na época da avaliação variou de 28 a $180 \mathrm{ng} / \mathrm{mL}$, sendo que 11 dos pacientes usavam bromocriptina. Detectou-se presença atual de distúrbios de ansiedade em 18 pacientes (56,2\%) e 5 controles (31,2\%), depressão em 10 pacientes $(31,2 \%)$ e 2 controles $(12,5 \%)$, distmia em duas pacientes e outros diagnósticos psiquiátricos em 6 pacientes $(18,7 \%)$. Os escores da depressão variaram entre 16 e 31 nos pacientes e foram 12 e 16 nos controles. A frequência de sintomas psiquiátricos como um todo, foi significativamente maior nos hiperprolactinêmicos (teste do quiquadrado), mas a diferença não foi significativa na análise isolada de ansiedade ou depressão. A hiperprolactinemia representa um fator de risco de 3,57 para depressão, 3,32 para ansiedade e 3,84 para outros sintomas psiquiátricos. Não houve diferença significativa na frequência de sintomas psiquiátricos entre portadores ou não de adenomas hipofisários e usuários ou não de bromocriptina. Não houve correlação $(r=0,07)$ entre a prolactina e a frequência de sintomas psiquiátricos. Conclui-se pela necessidade de atentar para a concomitância de hiperprolactinemia e distúrbios psiquiátricos, cujo reconhecimento permitirá abordagem terapêutica específica.
\end{abstract}

PALAVRAS-CHAVE: hiperprolactinemia, prolactinoma, ansiedade, depressão, bromocriptina.

\section{Hyperprolactinemia and psychological disturbance}

ABSTRACT - Psychological symptoms, specially anxiety and depression, have been associated to hyperprolactinemia. To evaluate the presence of these symptoms, 32 patients ( 5 men and 27 women) with hyperprolactinemia of several etiologies and 15 individuals with normal prolactin levels were submitted to the Composed International Diagnostic Interview, followed by the Hamilton rating scale for depression. The serum prolactin, at the time of evaluation, ranged between 28 and $180 \mathrm{ng} / \mathrm{mL}$. Eleven patients were receiving bromocriptine. The presence of anxiety was detected in 18 patients (56.2\%) and 5 controls (32.2\%), depression was detected in 10 patients (31.2\%) and 2 controls (12.5\%), dysthymia in 2 patients and other psychiatric diagnosis in 6 patients (18.7\%). The scores of depression ranged between 16 and 31 for the patients, and were 12 and 16 for the controls. The frequency of psychiatric symptoms, as a whole, was significantly higher in the hyperprolactinemic patients (chi-square test), but the difference was not significant in isolated analysis of anxiety and depression. The hyperprolactinemia represents a risk of 3.52 for depression, 3.32 for anxiety and 3.84 for other psychiatric symptoms. There was no significant difference in the frequency of psychiatric symptoms among patients with or without pituitary adenomas nor users or not users of bromocriptine. There was no correlation $(\mathrm{r}=0,07)$ between prolactin and the frequency of psychiatric symptoms. These results emphasize the importance of a special attention to the concomitance of hyperprolactinemia and psychiatric disturbance, which will allow a specific therapeutic approach.

KEY WORDS: hyperprolactinemia, prolactinoma, anxiety, depression, bromocriptine.

Disciplina de Endocrinologia da Fundação Faculdade Federal de Ciências Médicas (FFCM) Santa Casa de Misericórdia (SCM) de Porto Alegre (PA): *Doutora, Livre-Docente em Endocrinologia, Professora Adjunta da Disciplina de Endocrinologia da FFFCMPA e Chefe do Serviço de Endocrinologia da ISCMPA; *Médica Endocrinologista; ***Bolsista de iniciação científica. Aceite: 6-abril-2000.

Dra. Miriam da Costa Oliveira - Rua Dona Mimi Moro 40 - 90480-050 Porto Alegre RS - Brasil. Fax: 513286761. E-mail:mco@portoweb.com.br 
A hiperprolactinemia está classicamente associada a disfunção gonadal. No entanto, já em 1975, foi chamada a atenção para sua associação com sintomas psicológicos ${ }^{1}$. Ansiedade, somatização, hostilidade e/ou depressão foram, a partir de então, relacionadas com hiperprolactinemia ${ }^{2,3}$. São poucos os estudos publicados sobre o tema, em geral com número pequeno de pacientes. Esses estudos nem sempre excluem outras patologias relacionadas a distúrbios psicológicos, utilizam escalas variáveis e às vezes inadequadas de quantificação dos sintomas psiquiátricos e têm resultados discordantes quanto à relação entre os níveis prolactinêmicos e esses sintomas.

Tendo em vista a alta prevalência de hiperprolactinemia na população, da ordem de 1 a $10 \%{ }^{4}$ foi objetivo deste estudo explorar a relação de níveis elevados de prolactina com sintomas comportamentais, para ampliar o conhecimento em fisiopatologia psiconeuroendócrina e o papel de agonistas dopaminérgicos nesta área.

\section{MÉTODO}

Os pacientes hiperprolactinêmicos foram identificados através da listagem de indivíduos que dosaram prolactina no laboratório central de Santa Casa de Misericórdia de Porto Alegre. Esta listagem registra 1485 dosagens de prolactina entre janeiro de 1996 e março de 1998. As dosagens foram feitas por quimioluminescência, através de kits CHIRON Diagnostics, cuja faixa de normalidade varia , nas mulheres, entre 2,8 e 29,2 e, nos homens 2,1 e $17,7 \mathrm{ng} / \mathrm{ml}$, e com limite inferior de deteç̧ão de $0,3 \mathrm{ng} / \mathrm{ml}$. A dosagem foi realizada em amostra única de sangue ou em mistura de três amostras, segundo o pedido dos médicos solicitantes.

No período em estudo foi detectada hiperprolactinemia em 217 ocasiões, referentes a 162 diferentes indivíduos. Desses, foi possível contatar, através de carta ou através de consulta regular no ambulatório de endocrinologia da instituição, 59 pacientes, 33 dos quais consentiram em participar da pesquisa e se submeteram ao questionário psiquiátrico. Um desses casos foi excluído do protocolo ao se constatar hipotireoidismo durante a avaliação. Os pacientes previamente submetidos a cirurgia ou radioterapia hipofisária apresentavam adequada reposição hormonal. Nenhum caso apresentava outra doença endócrina relacionada a depressão.

O grupo controle foi formado por indivíduos normoprolactinêmicos identificados através da mesma listagem utilizada para os casos. Foram contatados, por carta, indivíduos pareados por sexo e faixa etária com os hiperprolactinêmicos. Foram entrevistados 16 controles de modo similar aos casos.

A entrevista foi feita através do Composed International Diagnostic Interview (CIDI) ${ }^{5}$, de validação internacional, que utiliza o Código Internacional de Doenças (CID 9) e o Diagnostic Statistical Manual (DSM-IIIR). A análise dos sintomas levou em conta o caráter temporal deles, isto é: sintomas atuais, os presentes nas últimas duas semanas; sintomas recentes, os ocorridos nos um a seis meses prévios; e, passados, os ocorridos há mais de seis meses. Os sintomas foram agrupados em distúrbios de ansiedade, depressão e outros diagnósticos psiquiátricos. Os distúrbios de ansiedade englobam agorafobia, fobia social, fobia isolada, ansiedade generalizada, pânico, hipocondria, transtorno obsessivo compulsivo e outras ansiedades. Os outros diagnósticos psiquiátricos incluem bulimia, convulsão dissociativa, dor somatoforme, amnésia dissociativa, desordem de movimento, perda sensorial, esquizofrenia, uso de álcool ou droga ilícita. Quando feito o diagnóstico de depressão, esta foi quantificada através da escala de Hamilton ${ }^{6}$. A aplicação dos questionários foi realizada por um dos autores (LG), médico anteriormente treinado por psiquiatra para este fim, e com experiência prévia na aplicação dos questionários.

Casos e controles tiveram seus prontuários revisados, originando uma planilha de dados clínicos e laboratoriais.

$\mathrm{Na}$ análise estatística foi utilizado o teste do qui-quadrado seguido ou não pelo teste exato de Fischer. A possível associação entre os níveis de prolactina e a presença de sintomas psicológicos, foi avaliada através da análise de regressão. Um nível de significância igual a 0,05 foi utilizado em todos os testes.

Este projeto foi aprovado pelo Comitê de Ética da instituição.

\section{RESULTADOS}

O número de pacientes hiperprolactinêmicos representou $11,3 \%$ da população que se submeteu à dosagem da prolactina sérica no laboratório citado.

\section{Caracterização da amostra}

O grupo de pacientes com hiperprolactinemia compreendeu 32 pacientes com idade média de $35,9 \pm 11,7$ anos: 5 homens ( 20 a 71 anos na entrevista) e 27 mulheres (15 a 50 anos na entrevista). 
Tabela 1. Causas de hiperprolactinemia na amostra.

\begin{tabular}{ll}
\hline Alterações hipotálamo-hipofisárias & 8 \\
Microadenomas & \\
Macroadenomas & 3 \\
$\quad$ Com hipofisectomia e radioterapia prévias & 1 \\
Com hipofisectomia & 2 \\
$\quad$ Sem tratamento ou com tratamento clínico & 1 \\
Interrupção da haste hipofisária (calcificação) & 1 \\
Radioterapia hipofisária & 2 \\
Drogas & 1 \\
Outras causas & 1 \\
Síndrome dos ovários policísticos & 1 \\
Síndrome dos ovários policísticos e sela vazia & 11 \\
Sela vazia e drogas & 32 \\
Idiopática & \\
Total &
\end{tabular}

Do ponto de vista clínico, 23 mulheres apresentavam distúrbio menstrual: amenorréia em 19 e irregularidade menstrual em 4; 19/23 apresentavam galactorréia; 8/10 mulheres relatavam diminuição da libido. Entre os homens, a diminuição da libido estava presente em 4/5.

Os níveis séricos da prolactina no período da avaliação variaram de 28 a $180 \mathrm{ng} / \mathrm{mL}$ (média $89,2 \pm 47,3$ ). Embora todos os pacientes se encontrassem hiperprolactinêmicos na época da aplicação do questionário, 11 deles estavam sob tratamento com bromocriptina, em doses variando entre $2,5 \mathrm{a}$ $10 \mathrm{mg} / \mathrm{dia}$.

Lesão compatível com adenoma hipofisário foi detectada através de tomografia computadorizada em 14/26 casos avaliados; em 8 ocasiões tratava-se de um microadenoma.

Quanto à etiologia da hiperprolactinemia (Tabela 1) predominaram as causas de origem central, com destaque para os adenomas hipofisários (43,7\% da amostra). Dos macroadenomas submetidos a ressecção cirúrgica $(n=4)$, em dois a imuno-histoquímica demonstrou a presença de células secretoras de prolactina e hormônio do crescimento e um mostrou-se não secretor. Em algumas situações a etiologia é dúbia, uma vez que detectou-se a presença concomitante de dois fatores classicamente associados à hiperprolactinemia. As drogas relacionadas ao aumento da prolactina sérica utilizadas pelos pacientes foram antidepressivos tricíclicos, neurolépticos e cocaína.

\section{Sintomas psicológicos}

Os resultados do questionário mostraram a presença atual e/ou recente de distúrbios de ansiedade em 18 pacientes (56,2\%, 2 homens e 16 mulheres), depressão em dez (31,2\%, 2 homens e 8 mulheres), distmia em 2 mulheres e outros diagnósticos psiquiátricos em 6 (18,7\%, 5 mulheres e um homem). Os distúrbios de ansiedade estão discriminados na Tabela 2 e os outros diagnósticos na Tabela 3.

No passado, foram detectados distúrbios de ansiedade em quatro pacientes, depressão em três e outros diagnósticos em 9. Um desses casos apresentou bulimia no passado. Apenas dois 
Tabela 2. Distúrbios de ansiedade atuais ou recentes ${ }^{l}$ na amostra (número de diagnósticos efetuados ${ }^{2}$ ).

\begin{tabular}{ll}
\hline Agorafobia & 8 \\
Fobia social & 8 \\
Fobia simples & 6 \\
Ansiedade generalizada & 5 \\
Pânico & 2 \\
Hipocondria & 1 \\
Transtorno obsessivo-compulsivo & 1 \\
Outras ansiedades & 5 \\
Total & 36 \\
\hline
\end{tabular}

${ }^{1}$ Recente: presente nos últimos 6 meses; atual: presente nas duas últimas semanas. ${ }^{2} \mathrm{O}$ diagnóstico foi estabelecido em 8 vezes através do CID, 5 vezes através do DMS e nas demais pelos dois questionários.
Tabela 3. Outros diagnósticos psiquiátricos atuais ou recentes ${ }^{l}$ na amostra (número de diagnósticos efetuados ${ }^{2}$.

\begin{tabular}{ll}
\hline Bulimia & 2 \\
Dor somatoforme & 2 \\
Amnésia dissociativa & 2 \\
Convulsão dissociativa & 1 \\
Perda sensorial & 1 \\
Uso de álcool & 1 \\
Total & 9 \\
\hline
\end{tabular}

${ }^{1}$ Recente: presente nos últimos 6 meses; atual: presente nas duas últimas semanas. ${ }^{2} \mathrm{O}$ diagnóstico foi estabelecido em 6 vezes através do CID e nas demais pelos dois questionários

desses pacientes não apresentam sintomas psiquiátricos atuais ou recentes (um deles apresentara desordem do movimento e outro, ansiedade generalizada).

Os escores da depressão variaram entre 16 e 31; dois desses pacientes estavam em uso de antidepressivos (fluoxetina, clomipramina, amitriptilina, imipramina) e um usara antidepressivo no passado (amitriptilina). Uma paciente com distmia relatava tentativa de suicídio no passado.

Entre os hiperprolactinêmicos, 6 (2 homens e 4 mulheres) eram tabagistas.

Em relação ao diagnóstico atual ou recente de sintomas psicológicos nos controles, 5 (31,2\%) apresentaram distúrbios de ansiedade (em 5 casos, fobia simples; em dois, ansiedade generalizada; em um agorafobia - dois desses diagnósticos foram estabelecidos pelo CID e dois pelo DSM); dois casos $(12,5 \%)$ apresentaram depressão, um deles com 12 pontos na escala de Hamilton e outro com 16; um indivíduo usava álcool. No passado, três tinham história de ansiedade, dois de depressão, um uso de maconha e três com outros diagnósticos psiquiátricos. Um dos controles era tabagista e nenhum usava antidepressivo.

A frequência dos sintomas psiquiátricos foi significativamente maior nos hiperprolactinêmicos em relação aos controles, mas não houve diferença significativa na frequência de ansiedade, depressão ou outros sintomas quando analisados separadamente.

A hiperprolactinemia, presente em 83,3\% dos indivíduos com depressão, presente em 78,3\% dos com distúrbios de ansiedade e em $85,7 \%$ dos com outros sintomas psiquiátricos, está associada a um fator de risco para a presença destes distúrbios de, respectivamente, 3,57, 3,32 e 3,84. O fator de risco para sintomas psiquiátricos no geral é de 3,49.

Entre os hiperprolactinêmicos com sintomas psiquiátricos, $31 \%$ apresentavam imagem compatível com adenoma hipofisário; a mesma imagem foi observada em $26 \%$ dos indivíduos sem sintomas psiquiátricos, não configurando diferença significativa entre esses dois grupos.

Dos indivíduos que usavam bromocriptina, 63,6\% apresentavam sintomas psiquiátricos. Não houve diferença significativa quanto à presença de sintomas psiquiátricos nos indivíduos usando ou não bromocriptina.

A análise de regressão entre os níveis de prolactina e a presença de sintomas psiquiátricos mostrou um coeficiente de correlação igual a 0,07 . 


\section{DISCUSSÃO}

A frequência de hiperprolactinemia $(11,3 \%)$ aqui detectada nos usuários de um laboratório de hospital geral, portanto uma amostra selecionada, foi discretamente superior aos valores descritos na população em geral ${ }^{4}$. O predomínio de hiperprolactinemia no sexo feminino, assim como o quadro clínico de síndrome galacto-amenorréia nas mulheres e diminuição da libido em ambos os sexos vão ao encontro dos dados de literatura.

A ausência de níveis acentuadamente elevados de prolactina na série, apesar da presença de prolactinomas, está possivelmente associada à abordagem cirúrgica prévia e ao uso de bromocriptina em vários casos. A falta de normalização dos níveis de prolactina na presença desse agonista dopaminérgico pode estar relacionada ao uso irregular deste ou à utilização de doses insuficientes para obtenção de valores normais de prolactina.

Há provas robustas dos efeitos da prolactina no comportamento em várias espécies animais? Associação de hiperprolactinemia com sintomas psiquiátricos está descrita na literatura. O primeiro sintoma descrito foi a ansiedade, antecedendo o início da irregularidade menstrual associada à hiperprolactinemia ${ }^{1}$. Outros autores confirmaram aumento de ansiedade e somatização na amenorréia hiperprolactinêmica ${ }^{2}$, assim como de hostilidade e depressão ${ }^{3}$. Em relação a controles, homens hiperprolactinêmicos também mostraram mais ansiedade ${ }^{8}$. Raramente, achados de hostilidade, ansiedade ou angústia não foram mais prevalentes em pacientes com aumento da prolactina ${ }^{9}$. No atual estudo, a presença de sintomas psiquiátricos foi significativamente maior nos hiperprolactinêmicos. Ansiedade e depressão ocorreram em valores percentuais aproximadamente duplicados em relação aos controles. A presença de hiperprolactinemia em aproximadamente $80 \%$ dos indivíduos com algum tipo de sintoma psiquiátrico, aqui detectada, eleva esta condição hormonal a um fator de risco para ocorrência de distúrbios emocionais superior a três vezes o da população em geral.

Sítios ligadores de prolactina no sistema nervoso central estão identificados no hipotálamo, plexo coróide e, em menor grau, na substância nigra. O papel da prolactina na patogênese dos distúrbios psiquiátricos pode refletir uma ação direta do hormônio no sistema nervoso central, um efeito indireto, através de outros hormônios gonadais ou adrenais, ou constituírem fatores independentes, resultantes da depleção de dopamina ${ }^{10}$.

Alguns viézes poderiam interferir na inter-relação sintomas psiquiátricos/hiperprolactinemia, como a presença de hipogonadismo associado ao aumento da prolactina. Disfunção sexual está presente em $58 \%$ dos hiperprolactinêmicos, mais noticeada nos homens. É difícil dissociar efeito comportamental direto da prolactina das consequências do hipogonadismo ${ }^{11}$.

A simples informação a um paciente da presença de um tumor hipofisário também poderia gerar ansiedade ou depressão, dependendo da personalidade do paciente e do médico, e do contexto cultural ${ }^{11}$. Na atual amostra, a similaridade de achados entre os portadores ou não de adenomas não confirma esta hipótese. Outros autores observaram mais ansiedade nas pacientes sem evidências de adenomas $^{12,13}$. Avaliando por outro prisma a mesma questão, Reavley e colaboradores observaram ansiedade em $54 \%$ de hiperprolactinêmicos, contra $27 \%$ em um grupo controle, constituído por portadores de outros tumores hipofisários; depressão esteve presente em $25 \%$ das hiperprolactinêmicas contra $27 \%$ dos controles $^{13}$.

Depressão e diminuição da libido podem estar presentes de forma independente na hiperprolactinemia, sendo indefinida a relação entre esses achados, uma vez que a diminuição da libido pode resultar da hiperprolactinemia ou da depressão, ou ser causa da depressão ${ }^{14}$.

Há relatos de diminuição da angústia e aumento do bem-estar com a redução dos níveis da prolactina secundariamente ao uso da bromocriptina ${ }^{10}$, assim como melhora da depressão com bromocriptina ou pergolide ${ }^{15}$ e melhora do bem-estar com quinagolide ${ }^{16}$. No entanto, constatando-se ausência de correlação entre a melhora nos escores da depressão e o grau de supressão da prolactina, foi sugerido que a bromocriptina pudesse ter efeitos independentes sobre a secreção hormonal e a 
depressão ${ }^{9}$. Em pacientes deprimidos, sem aumento da prolactina, a bromocriptina já havia apresentado efeitos antidepressivos similares aos da imipramina ${ }^{17}$. Sitland-Marken e colaboradores, revisando publicações onde a bromocriptina foi utilizada para o tratamento da depressão, observaram resultados positivos em todos os estudos ${ }^{18}$. Nossos resultados não confirmaram efeito antidepressivo intrínseco da bromocriptina, uma vez que os achados não foram diferentes nos grupos com ou sem a droga.

Por outro lado, não consideramos aqui a alternativa literalmente oposta aos argumentos até então citados, de que a bromocriptina poderia ser responsável por sintomas mentais como alucinações, confusão ou excitação psicomotora. Estes efeitos, raros, são dose-dependentes e observados em indivíduos mais velhos, em geral com história prévia de doença psiquiátrica ${ }^{19}$. Ao mesmo tempo, os sintomas de ansiedade aqui detectados foram de intensidade distinta dos associados ao uso de levodopa e drogas correlacionadas.

Embora tenha sido observada correlação entre a concentração de prolactina e os escores psicológicos ${ }^{15}$, este achado não foi aqui confirmado, como também não o foi por Lappöhn e colaboradores ${ }^{16}$.

Um último dado a chamar a atenção é a presença de bulimia nervosa, atual ou passada, em três casos desta série. Uma possível relação entre hiperprolactinemia e bulimia, do conhecimento dos autores, não está descrita na literatura e merece investigação futura.

Uma vez detectada a alta prevalência de distúrbios psiquiátricos em pacientes hiperprolactinêmicos, conclui-se pela necessidade de investigação cuidadosa tanto de indivíduos com queixas psiquiátricas, quanto de indivíduos com hiperprolactinemia, com a finalidade de detectar situações potencialmente tratáveis e que permitam melhor qualidade de vida para estes pacientes. Conclui-se também pela necessidade de estudos que avaliem o efeito do tratamento da hiperprolactinemia sobre as manifestações psiquiátricas e estudos que investiguem as bases neuropatológicas desta associação.

\section{REFERÊNCIAS}

1. Tyson JE, Andreasson B, Huth J, Smith B, Zacur H. Neuroendocrine dysfunction in galactorrhea-amenorrhea after oral contraceptive use. Obstet Gynecol 1975;46:1-11.

2. Fava GA, Fava M, Kellner R, Serafini E, Mastrogiacomo I. Depression, hostility and anxiety in hyperprolactinemic amenorrhea. Psychother Psychosom 1981;36:122-128.

3. Kellner R, Buckman MT, Fava GA, Pathak D. Hyperprolactinemia, distress, and hostility. Am J Psychiatry 1984;141:759-763.

4. Molitch ME: Prolactin. In Melmed S (ed). The pituitary. Cambridge: Blackwell Science, 1995:136-186.

5. World Health Organization: The Composed International Diagnostic Interview (CIDI). Geneva: World Health Organization, 1990.

6. Hamilton M. A rating scale for depression. J Neurol Neurosurg Psychiatry 1960;23:56.

7. Dutt A, Kaplitt MG, Kow L-M, Pfaff DW. Prolactin, central nervous system and behavior: a critical review. Neuroendocrinology 1994;59:413-419.

8. Fava M, Fava GA, Kellner R, Serafini E, Mastrogiacomo I. Psychological correlates of hyperprolactinemia in males. Psychother Psychosom 1982;37:214-217.

9. Koppelman MCS, Parry BL, Hamilton JA, Alagna SW, Loriaux DL. Effect of bromocriptine on affect and libido in hyperprolactinemia. Am J Psychiatry 1987;144:1037-1041.

10. Buckman MT, Kellner R. Reduction of distress in hyperprolactinemia with bromocriptine. Am J Psychiatry 1985;142:242-244.

11. Sobrinho LG. Emotional aspects of hyperprolactinemia. Psychother Psychosom 1998;67:133-139.

12. Keller SK, Neuhaus-Theil A, Quabbe HJ. Psychological correlates of prolactin secretion: aggression and depression. Acta Endocrinol 1985;108:118-119.

13. Reavley A, Fischer AD, Owen D, Creed FH, Davis JR. Psychological distress in patients with hyperprolactinaemia. Clin Endocrinol 1997;47:343-348.

14. Fava M, Fava GA, Kellner R. Psychosomatic aspects of hyperprolactinemia. Psychother Psychosom 1983;40:257-262.

15. Mattox JH, Buckman MT, Bernstein J, Pathak D, Kellner R. Dopamine agonists for reducing depression associated with hyperprolactinemia. J Reprod Med 1986;31:694-698.

16. Lappöhn RE, van de Wiel HBM, Brownell J. The effect of two dopaminergic drugs on menstrual function and psychological state in hyperprolactinemia. Fertil Steril 1992;58:321-327.

17. Teohar C, Fischer-Cornelssen K, Akesson HO. Bromocriptine as antidepressant: double-blind comparative study with imipramine in psychogenic and endogenous depression. Curr Ther Res 1981;30:830-842.

18. Sitland-Marken PA, Wells BG, Froemming JH, Chu CC, Brown CS. J Clin Psychiatry 1990;51:68-82.

19. Sarne DH. Medical treatment of pituitary tumors and hypersecretory states. In: Becker KL (ed). Principles and practice of endocrinology and metabolism. Philadelphia: JB Lippincott, 1995:223-229. 